Dom. Cien., ISSN: 2477-8818

Vol. 4, núm. 1, enero, 2018, pp. 102-114

Desempeño docente en la capacitación del acompañamiento al examen complexivo

\title{
Desempeño docente en la capacitación del acompañamiento al examen complexivo
}

\section{Teaching performance in the accompaniment training for complexive exam}

\section{Desempenhar o documento na capacitação do acompañamiento do exame complexo}

Sebastián G. Yánez-Segovia I

sgyanez@uce.edu.ec

Herman W. Hernández-Benalcázar ${ }^{\mathrm{II}}$

hhernandez@uce.edu.ec

Lisbeth A. Cheza-Rodríguez III

lisalejandra28@hotmail.com

Washington R. Valdiviezo-Leroux ${ }^{\text {IV }}$

rvaldiviezoleroux@yahoo.com
Jhony F. Méndez-Játiva $v$

jmendez@uce.edu.ec

Mario A. Rivera-Valenzuela VI mariverav@uce.edu.ec

Carlos A. Vargas-Cumbajín VII cvargas@uce.edu.ec

Recibido: 20 de octubre de 2017 * Corregido: 20 de noviembre de 2017 * Aceptado: 01 enero de 2018

Ingeniero Agrónomo, Docente de la Universidad Central del Ecuador, Quito, Ecuador.

Biólogo, Docente de la Universidad Central del Ecuador, Quito, Ecuador.

Estudiante, de la Universidad Central del Ecuador, Quito, Ecuador.

Economista, Docente de la Universidad Central del Ecuador, Quito, Ecuador.

Ingeniero Agroindustrial, Docente de la Universidad Central del Ecuador, Quito, Ecuador.

Ingeniero Mecánico, Docente de la Universidad Central del Ecuador, Quito, Ecuador.

Biólogo, Docente de la Universidad Central del Ecuador, Quito, Ecuador. 


\section{Resumen}

En este artículo se estudió el desempeño de los docentes en el acompañamiento al examen complexivo de los estudiantes egresados de la Facultad de Ciencias Agrícolas de la Universidad Central del Ecuador. Mediante el análisis de las estrategias, motivos y enfoques de aprendizaje que modelan el cometido del docente e influyen en su desempeño. La falta de interés y los documentos publicados alrededor de las Ciencias Agrícolas dan énfasis a la investigación experimental, dejando relegada la investigación educativa, dando como resultado pocos estudios sobre el tema a nivel nacional e internacional. Se aplicó en forma voluntaria el Cuestionario de Procesos de Estudio Revisado. La población estuvo conformada por treinta y dos estudiantes de la Carrera de Ingeniería Agronómica y tres docentes de diversas especialidades profesionalizantes. Los resultados muestran que la mayoría de estudiantes prefieren el enfoque superficial, que tiene como base fundamental la motivación extrínseca, y apuesta por el aprendizaje memorístico. No se encontró ninguna relación relevante entre el enfoque de aprendizaje y los factores extracurriculares. Se concluye que es fundamental conocer el desempeño de los docentes en el acompañamiento al examen complexivo, con la finalidad de implementar métodos y metodologías para conseguir el aprendizaje participativo y optimizar el recurso postulante.

Palabras clave: Profesión, directriz, metodologías. 


\section{Abstract}

In this article the performance of the teachers in the accompaniment to the complexsive exam of the students graduated from the Faculty of Agricultural Sciences of the Central University of Ecuador was studied. Through the analysis of strategies, motives and learning approaches that shape the role of the teacher and influence their performance. The lack of interest and the documents published around the Agricultural Sciences give emphasis to experimental research, leaving educational research relegated, resulting in few studies on the subject at national and international level. The Study Process Questionnaire - Revised was applied in voluntary way. The population was confirmed by thirty two students of Agronomic Engineering and three teachers of diverse professional specialties. The results show that the majority of students prefer the superficial approach, which is based on extrinsic motivation, and commits to rote learning. No relevant relationship was found between the learning approach and the extracurricular factors. It is concluded that it is essential to know the performance of teachers in the accompaniment to the complex examination, in order to implement methods and methodologies to achieve participatory learning and optimize the applicant resource.

Key words: Profession, guideline, methodologies. 


\section{Introducción.}

Las políticas públicas en América Latina acordes al sector de la educación están enfocadas en cómo mejorar el desempeño de los docentes. Los diagnósticos coinciden en señalar que las propuestas tradicionales ya no alcanzan. Pero hay también fuertes evidencias de que no es simple determinar cuáles son los cambios adecuados y mucho menos ponerlos en práctica. Los maestros son parte del problema en virtud de su falta de preparación, del corporativismo, del acomodarse a una carrera que expulsa a los mejores. Pero deben ser parte de la solución porque sin la participación, compromiso y dedicación de los profesores será imposible superar la desigualdad educacional. Es necesario poner a aprender a la fuerza laboral docente latinoamericana y a enseñar a alumnos que desafian a la pedagogía de la uniformidad (Namo de Mello, 2005).

La responsabilidad del docente en la dirección del proceso formativo, en la creación de condiciones favorables para el aprendizaje, y por su influencia en que podría tener en el alumnado con el cual desempeña su trabajo, ha identificado las características de los docentes; lo que ha permitido plantear propuestas que podrían repercutir en la mejora de la calidad del aprendizaje y el rendimiento académico, minimizando el fracaso escolar. Los enfoques de aprendizaje influyen en el rendimiento académico (Riveros et al., 2011; Gargallo et al., 2012; Mirete et al., 2015).

Los mecanismos de evaluación del desempeño de maestros y profesores constituyen uno de los aspectos claves en la gestión de la docencia. El interés por la calidad de dichos dispositivos ha crecido enormemente en los años recientes, porque permiten monitorear y fortalecer el desarrollo profesional docente. En el pasado, la evaluación docente no era considerada una actividad demasiado relevante; quienes enseñaban se encontraban más allá de cualquier tipo de cuestionamiento (Vaillant, 2008). 
Salim y Lotti (2011), aseguran que, "la presencia de la pluralidad en términos de capacidades, intereses o motivaciones para aprender, demanda una enseñanza centrada en el aprendizaje. Esto exige considerar al alumno y tener en cuenta cuáles son sus procesos de aprendizaje al momento de diseñar e implementar estrategias de enseñanza”.

El estudiante no es el único actor en el contexto educativo, pues el aprendizaje al ser una actividad cognitiva no puede separarse del medio cultural. A pesar de esto el estudiante es al final quien decide, haciendo del aprendizaje una actividad individual, dependiente de las características personales y recursos disponibles. De esta individualización del aprendizaje, Barca (2002), cita, que “el aprendizaje resulta de la interrelación de tres elementos claves: la intención (motivo) de quien aprende, las formas o procedimientos que utiliza (estrategia) y los logros que obtiene (rendimiento)".

El enfoque de aprendizaje no se constituye como una característica que el individuo posea, si no que se considera fruto de la interacción entre el individuo y su contexto de aprendizaje; por lo tanto no es una característica estática o inamovible del sujeto (García, 2016); es decir, el desarrollo de un determinado enfoque de aprendizaje se relaciona con las experiencias educativas vividas por el alumno y el docente; además posee, una naturaleza contextual. Las posibilidades de innovación dependen de los docentes y de las restricciones o potencialidades de los ámbitos institucionales específicos donde esas prácticas se desarrollan (Araujo, 2016).

Los profesores pueden potenciar el enfoque profundo del aprendizaje usando metodologías de enseñanza y evaluaciones pertinentes (Gargallo et al., 2012). El aprender requiere del interés y disposición del aprendiz por su propio aprendizaje o por las actividades que lo llevan a él (motivación), del uso de las habilidades, acciones y pensamientos que ocurren durante el aprendizaje 
(estrategias), y también qué concepción de aprendizaje mantiene el estudiante y cómo lo aborda (enfoques de aprendizaje) (Salim y Lotti, 2011).

Los modelos de gestión institucional de la docencia deberían orientar el desempeño de maestros y profesores, que no se sustenta a menudo en sistemas de evaluación sistemáticos y objetivos. El sistema de acompañamiento se basa, con frecuencia, en el antiguo esquema de inspección, cuyo propósito es mucho más burocrático que técnico. Hace falta un verdadero respaldo institucional que retroalimente los procesos de transformación, y ajuste el rol y las distintas tareas que deben afrontar tanto maestros como profesores en el ejercicio profesional (Vaillant, 2008).

La elaboración de criterios profesionales representa un esfuerzo por describir de manera observable lo que los docentes deben saber y ser capaces de hacer en el ejercicio de su profesión. Estos marcos intentan capturar el consenso de la investigación y de los docentes acerca de los conocimientos, capacidades y competencias que deben dominar en el acompañamiento del examen complexivo, con la finalidad de implementar métodos y metodologías del proceso de aprendizaje.

\section{Metodología.}

Se analizó las estrategias, motivos y enfoques de aprendizaje que modelan la percepción del estudiante egresado de la Carrera de Ingeniería Agronómica de la Facultad de Ciencias Agrícolas. Se aplicó en forma presencial, sin identificación alguna, la Encuesta establecida para Evaluación del Desempeño Docente, en su última versión establecida por Comisión de Evaluación Interna de la Universidad Central del Ecuador. La población estuvo conformada por todos los estudiantes que asistieron a la capacitación de Acompañamiento para el examen Complexivo. Se relacionaron los enfoques académico (1), ordenamiento / humanísticas (2), andragogía (3) y dominio profesional (4) 
(Tabla 1). La opinión de los referidos señores estudiantes es objetiva, ya que no han sido educandos de los capacitadores durante su carrera de pre grado, con la finalidad de implementar métodos y metodologías para conseguir el aprendizaje significativo.

Tabla 1. Cuestionario empleado en el estudio del desempeño docente en el acompañamiento al examen complexivo de estudiantes egresados en la Carrera de Ingeniería Agronómica de la Facultad de Ciencias Agrícolas en la Universidad Central del Ecuador.

\begin{tabular}{|c|c|c|}
\hline ÍTEMS & PREGUNTAS & TIPO DE VARIABLE \\
\hline 1 & $\begin{array}{l}\text { Cumplió con la programación del contenido de las clases de } \\
\text { acompañamiento al examen complexivo }\end{array}$ & ACADEMICO \\
\hline 2 & $\begin{array}{l}\text { Informo sobre los propósitos y horarios de los temas en las clases de } \\
\text { acompañamiento al examen complexivo }\end{array}$ & ACADEMICO \\
\hline 3 & Cumplió el horario de clases establecido & ACADEMICO \\
\hline 4 & Registro la asistencia de los estudiantes en cada clase & ACADEMICO \\
\hline 5 & Se relacionó con los estudiantes con respecto dentro y fuera del aula & ORDENAMIENTO / HUMANISTICAS \\
\hline 6 & Propicio el dialogo en el desarrollo de clases & ORDENAMIENTO / HUMANISTICAS \\
\hline 7 & Genero un ambiente de confianza en el aula & ORDENAMIENTO / HUMANISTICAS \\
\hline 8 & Fomento equidad de género en las actividades académicas & ORDENAMIENTO / HUMANISTICAS \\
\hline 9 & Demostró que sus clases fueron planificadas y no improvisadas & DOMINIO CIENTIFICO \\
\hline 10 & Demostró dominio de la asignatura & DOMINIO CIENTIFICO \\
\hline 11 & Desarrollo la asignatura utilizando diferentes estrategias metodológicas & ANDRAGOGIA \\
\hline 12 & Mantuvo el interés del estudiante durante el trabajo académico & ORDENAMIENTO / HUMANISTICAS \\
\hline 13 & Promovió actividades participativas durante el proceso de inter aprendizaje & ANDRAGOGIA \\
\hline 14 & Utilizo la investigación para construir y reconstruir los aprendizajes & DOMINIO CIENTIFICO \\
\hline 15 & Fomento el trabajo en equipo & ACADEMICO \\
\hline 16 & Utilizo la TICs, como recurso didáctico en el trabajo académico & ACADEMICO \\
\hline 17 & Desarrollo los valores en base al ejemplo & ORDENAMIENTO / HUMANISTICAS \\
\hline 18 & Evito todo tipo de discrimen religioso, ético, político y de genero & ORDENAMIENTO / HUMANISTICAS \\
\hline 19 & Promovió buenas relaciones interpersonales en el ambiente académico & ORDENAMIENTO / HUMANISTICAS \\
\hline 20 & Satisfizo sus expectativas la labor del docente & DOMINIO CIENTIFICO \\
\hline 21 & Contribuyo en su crecimiento personal y profesional la labor del docente & DOMINIO CIENTIFICO \\
\hline
\end{tabular}


Se realizó un análisis paramétrico de las preguntas (ítems), formuladas en el respectivo cuestionario.

\section{Resultados y discusión.}

Existen resultados perceptibles del empoderamiento de aprendizaje y conocimiento de los alumnos que fueron capacitados en el acompañamiento al examen complexivo. El procesamiento de datos demostró tres diferentes respuestas en cuanto a la Evaluación del Desempeño Docente, presentándose en los tres docentes evaluados calificación superior al 85\%, con una respuesta excelente en cuanto a la ponderación de datos establecidos en evaluaciones docentes universitarias nacionales e internacionales (Figura 1).

La evaluación del docente es un proceso que requiere una precisa definición en sus modelos de partida, así como una adecuada sistematización a través de la investigación, para que pueda ser entendida como un dispositivo de aprendizaje profesional y organizacional que permita orientar y fortalecer la actividad docente, contribuyendo al mejoramiento de los aprendizajes alcanzados por los estudiantes (Álvarez, 1997).

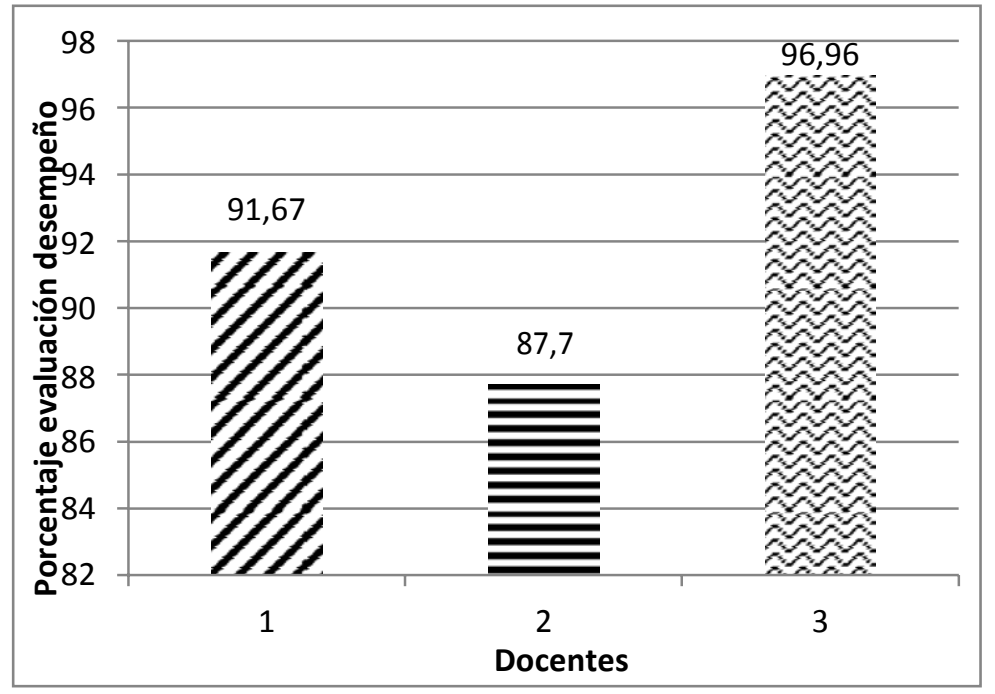

Figura 1. Respuestas consolidadas a la hetero evaluación del desempeño Docente 
El enfoque desde el cual se evalúa la actividad docente depende de la concepción teórica de partida, y del propósito que persiga la evaluación. En este sentido, la literatura identifica diversos modelos de evaluación posibles basados en el perfil del docente; en los resultados obtenidos por los estudiantes; en los comportamientos del aula; y en las prácticas reflexivas (Darling, 1998).

La utilización de pruebas, tanto durante la formación inicial como a lo largo de la carrera docente, ha sido objeto de controversia y debate en los Estados Unidos. Varios estudios realizados sobre el tema llegan a la conclusión que las preguntas incluidas en los tests suelen ser de baja calidad (Shanker, 1996; Darling, 1998), que faltan criterios de validez y fiabilidad en la mayor parte de las pruebas (Dybdahl et al., 1997), y que los resultados de los tests se establecen en función de criterios de ordenamiento de puntajes y no sobre la base de criterios referidos a la calidad de la enseñanza impartida.

Ingvarson (1998), afirma en sus artículos e investigaciones que si pretendemos que los esquemas de evaluación docente puedan mejorar los sistemas educacionales, entonces se vuelve imprescindible modificar paralelamente las estructuras de la carrera docente. Muchas veces la evaluación se concibe como un instrumento de rendición de cuentas para los administradores. Este tipo de evaluación es necesaria pero no suficiente; se requieren también modelos profesionales de responsabilidad que en buena medida dependen de la capacidad de definir estándares profesionales.

El mecanismo de evaluación docente en Uruguay es muy similar al de Argentina y Nicaragua, ya que los maestros son evaluados por sus directores y supervisores. La evaluación de los directores es anual y refiere fundamentalmente al desempeño del docente en el centro educativo y con la comunidad. La evaluación de los supervisores, en cambio, se concentra en la labor en el aula, priorizando los aspectos didácticos. El supervisor visita a cada maestro dos veces por año, 
como mínimo. El director y el supervisor califican al maestro en una escala de cinco tramos. Esta calificación se combina con otros indicadores como antigüedad y actividad computada, que es el cociente entre los días trabajados y los días hábiles que el docente debería haber trabajado. El índice resultante es la antigüedad calificada. El puntaje que el docente recibe es avalado por una junta calificadora integrada por tres miembros y es sobre él que se realiza el ordenamiento de los docentes dentro de cada grado (Vaillant, 2008).

En cuanto al análisis de las preguntas, los resultados muestran que los estudiantes en las variables tipo 2, 3 y 4 muestran una clara acogida al desempeño de los docentes capacitadores; por otra parte, en lo referente a la pregunta número quince, correspondiente a la variable académica, que se refiere al "Fomento de trabajo en equipo", presenta la menor respuesta numérica, determinada por el $78,70 \%$ de acuerdo a la exigencia que se estableció a este análisis, lo que nos muestra que podemos mejorar en este particular académico (Figura 2).

La hetero evaluación nos presenta la percepción real de los estudiantes egresados que tienen visión externa de los conocimientos impartidos. De los resultados a los estudiantes capacitados se puede apreciar adecuado acompañamiento andragógico; además se constató que los conocimientos son bien recibidos.

El TVAAS (Tennessee Value Added Assessment System) tiene por objetivo evaluar los aportes del distrito, la escuela y el docente en la formación del estudiante, mediante la aplicación de tests anuales. Este sistema de evaluación relaciona la acreditación lograda por el docente en su desempeño, con los avances de los estudiantes en sus aprendizajes (Carey, 2004). El caso de Texas constituye un buen ejemplo de la evolución que se registra durante los últimos años. En 1986 se puso en marcha el Texas Examination of Current Administrators and Teachers (TECAT), que 
consistía en una prueba destinada a evaluar conocimientos básicos sobre la enseñanza y gestión de centros educativos. Los índices de aprobación fueron elevados. Sin embargo, las investigaciones sobre el tema dejaron en evidencia que el TECAT adolecía de una serie de debilidades referidas, entre otros aspectos, a los altos costos de implementación, los escasos datos disponibles para apreciar la calidad de la enseñanza impartida, e incluso el stress que generaba en los docentes su propia aplicación. Es así que el TECAT se suprime, siguiendo la evolución de los años 90, y se remplaza por nuevas normas y requisitos (EXCET) que establecen los conocimientos y competencias que un docente debe poseer en cada etapa de su ejercicio profesional. Una evaluación anual basada en un plan de desarrollo profesional de cada docente se convierte en un requisito para la renovación del cargo (Vaillant y Rossel, 2006).

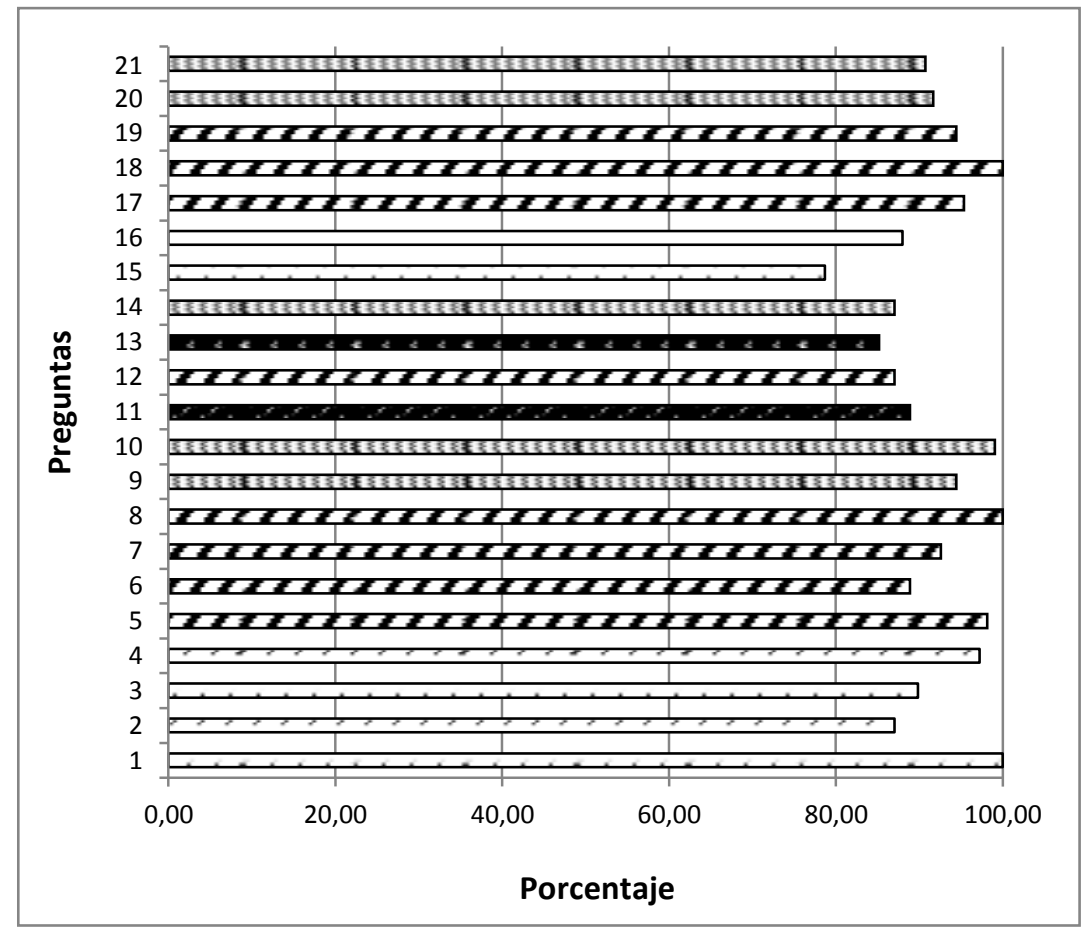

Figura 2. Respuestas consolidadas al enfoque de la percepción objetiva por Item evaluado. 
En los resultados, se resalta que es fundamental conocer el desempeño docente con capacitación a estudiantes egresados de nuestra Carrera en las clases de acompañamiento para el Examen Complexivo. Todas estas evaluaciones son elementos de mejoramiento por parte del docente capacitador, con lo que nos ayudara a fortalecer la labor de aprendizaje.

Al evaluador, le interesará indagar sobre la formación de base y la experiencia del docente, o sobre los vínculos que establece con su alumnado y con el saber, o sobre las estrategias que utiliza para promover la motivación en sus estudiantes, o sobre el tipo de vínculo que cultiva con sus colegas, para citar tan solo algunos ejemplos de las posibles aproximaciones al tema (Darling, 1998).

Las respuestas provenientes de la presente evaluación se pueden interpolar para delimitar con certeza el fracaso y la deserción estudiantil. Navarro (2003), señala, que al analizar la actitud docente ante la evaluación, afirma que "prevalece un sentido general de insatisfacción en cuanto a la capacidad que los sistemas educativos han tenido para utilizar los resultados de estas evaluaciones de manera efectiva en, por un lado, orientar políticas y reformas nacionales, pero, muy especialmente, para llegar a la escuela y el aula de forma constructiva y práctica”

\section{Bibliografía.}

Álvarez, F. (1997). Evaluación de la acción docente en Latinoamérica. Santiago de Chile: Programa de Promoción de la Reforma Educativa en América Latina PREAL.

Araujo, S. (2016). Tradiciones de enseñanza, enfoques de aprendizaje y evaluación: dos puntos de vista, dos modos de actuación. Trayectorias Universitarias, 2(2):3-10. http://revistas.unlp.edu.ar/TrayectoriasUniversitarias

Barca, A. (2002). Los Contextos de Aprendizaje y Desarrollo en la Educación Secundaria Obligatoria. Perspectivas de Intervención Psicoeducativa sobre el fracaso escolar en La comunidad autónoma de Galicia Proyecto Feder/Esog-Galicia: 1fd97-0283. Universidad de la Coruña. España. 
Carey, K. (2004). The real value of teachers. A Publication of The Education Trust, 8(1).

Darling, L. (1998). Teachers and Teaching: Testing Policy Hypotheses From a National Commission Report. Educational Researcher, 27(1), pp. 5-15.

Dybdahl, C.; Shaw, D.; Edwards, D. (1997). Teachers Testing: Reasons for Rhetoric. Journal of Research and Development in Education 30(4), pp. 248-254

García, M. (2016). ¿Cómo aprenden los alumnos en su primer año de universidad al inicio y al final del curso? REDU. Revista de Docencia Universitaria, 14(1): 27-49.

Gargallo, B.; Suárez, J.; García, E.; Pérez, C.; Sahuquillo, P. (2012). Enfoques de aprendizaje en estudiantes universitarios excelentes y en estudiantes medios. Revista Española de Pedagogía, 70(252): $185-200$.

Ingvarson, L. (1998). Professional development as the pursuit of professional standards: the standardsbased professional development system. Teaching and Teacher Education, 14(1), pp. 127-140.

Mirete, A.; Soro, M., Maquilón, J. (2015). El fracaso escolar y los enfoques de aprendizaje: medidas para la inclusión educativa. Revista Electrónica Interuniversitaria de Formación del Profesorado, 18(3), 183-196. DOI: http://dx.doi.org/10.6018/reifop.18.3.239021

Namo de Mello, G. (2005). Profesores para la igualdad educacional en América Latina. Calidad y nadie de menos. Revista PREALC, 1.

Navarro, J. (2003). La evaluación y las actitudes de los docentes frente a ella: dificultades y alternativas de políticas. Buenos Aires: IIPE-UNESCO.

Riveros, E.; Bernal, M.; Gonzalez, N. (2011). Prevalencia de los enfoques de aprendizaje en estudiantes de Fisiología Médica: cuestionario de proceso de estudio revisado de dos factores (R-SPQ-2F). Biosalud, 10(2): 37-47.

Salim, R.; Lotti de Santos, M. (2011). Evaluación de enfoques, motivaciones y estrategias de aprendizaje en estudiantes del primer año universitario de Odontología (UNT). Cuadernos de Educación, 9: 245-260.

Shanker, A. (1996). Quality Assurance: what must be done to strengthen the teaching profession. Phi Delta Kappan 78: pp. 220-224

Vaillant, D.; Rossel, C. (2006). Docentes en Latinoamérica hacia una radiografía de la profesión. Santiago de Chile: PREAL.

Vaillant, D. (2008). Algunos marcos referenciales para la evaluación del desempeño docente en América Latina. Revista Iberoamericana de Evaluación Educativa, 1(2): 7-22 